\begin{tabular}{|c|c|c|c|c|c|c|}
\hline \multirow{4}{*}{ Impact Factor: } & ISRA (India) & $=3.117$ & SIS (USA) & $=0.912$ & ICV (Poland) & $=6.630$ \\
\hline & ISI (Dubai, UAE & $=0.829$ & РИНЦ (Russia) & $=0.156$ & PIF (India) & $=1.940$ \\
\hline & GIF (Australia) & $=0.564$ & ESJI $(\mathrm{KZ})$ & $=8.716$ & IBI (India) & $=4.260$ \\
\hline & JIF & $=1.500$ & SJIF (Morocco) & $=5.667$ & OAJI (USA) & $=0.350$ \\
\hline
\end{tabular}

\section{SOI: $1.1 /$ TAS $\quad$ DOI: $10.15863 /$ TAS \\ International Scientific Journal Theoretical \& Applied Science}

p-ISSN: 2308-4944 (print) e-ISSN: 2409-0085 (online)

Year: 2019 Issue: $07 \quad$ Volume: 75

Published: $23.07 .2019 \quad$ http://T-Science.org
QR - Issue

QR - Article
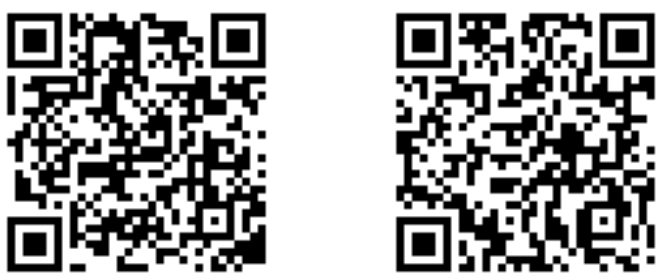

Vadim Andreevich Kozhevnikov

Peter the Great St.Petersburg Polytechnic University

Senior Lecturer

vadim.kozhevnikov@gmail.com

Maxim Yurievich Grigorev

Peter the Great St.Petersburg Polytechnic University

student

gri.jmax@gmail.com

\title{
THE STUDY OF MIGRATION FROM MYSQL TO NEWSQL DBMS TARANTOOL
}

\begin{abstract}
This work presents a description of the complete migration from MySQL to NewSQL Tarantool DBMS using the certain pattern of data base structure. Also we have given the general conception and description of each $D B M S$, reviewed analogues among DBMS. We have implemented specific schemes and functions for working with a database using both technologies. Based on comparative analysis of the speed of work conclusions were made about the effectiveness.

Key words: migration, MySQL, Tarantool, NewSQL, database design, Lua.

Language: Russian

Citation: Kozhevnikov, V. A., \& Grigorev, M. Y. (2019). The study of migration from MySQL to NewSQL DBMS Tarantool. ISJ Theoretical \& Applied Science, 07 (75), 243-259.

Soi: http://s-o-i.org/1.1/TAS-07-75-40 Doi: crossef https://dx.doi.org/10.15863/TAS.2019.07.75.40

Classifiers: database.

ИССЛЕДОВАНИЕ МИГРАЦИИ С СУБД MYSQL НА NEWSQL СУБД TARANTOOL

Аннотация: В данной работе изложено описание полной миграции с СУБД MySQL на NewSQL Tarantool на примере конкретной схемы базы данных. Даны общие понятия и описание каждой из СУБД. Рассмотрены аналоги на рынке СУБД. Реализованы конкретные схемы и функции для работы с базой данных с использованием обеих технологий. Проведен сравнительный анализ скорости работы и сделаны выводы об эффективности.

Ключевые слова: миграция, MySQL, Tarantool, NewSQL, проектирование базы данных, Lua
\end{abstract}

\section{Введение}

Ежедневно человечество генерирует колоссальный объём данных, но для того, чтобы эти данные имели какую-то ценность, и чтобы с ними можно было работать, они должны быть правильно обработаны. Обработанные данные называются информацией. В свою очередь для информации должно существовать хранилище, и в качестве этого хранилища выступают базы данных. С 1980-х годов базы данных, основанные на реляционной модели, занимают лидирующее положение среди прочих средств хранения информации.

С течением времени на рынке стали появляться конкуренты, а также новые технологические решения, и одним из таких решений стали NoSQL базы данных. Многие организации, которые ранее использовали реляционное решение, начали переходить на NoSQL решения. Для миграции данных существует ряд причин [1]:

- базы данных NoSQL зачастую предлагают компромисс, смягчая жесткие 


\begin{tabular}{|c|c|c|c|c|c|c|}
\hline \multirow{4}{*}{ Impact Factor: } & ISRA (India) & $=3.117$ & SIS (USA) & $=0.912$ & ICV (Poland) & $=6.630$ \\
\hline & ISI (Dubai, UAE & $=0.829$ & РИНЦ (Russia & $=0.156$ & PIF (India) & $=1.940$ \\
\hline & GIF (Australia) & $=0.564$ & ESJI (KZ) & $=8.716$ & IBI (India) & $=4.260$ \\
\hline & JIF & $=1.500$ & SJIF (Morocce & $=5.667$ & OAJI (USA) & $=0.350$ \\
\hline
\end{tabular}

требования свойств ACID ради более гибкой модели данных, которая допускает горизонтальное масштабирование. Благодаря этому NoSQL СУБД - отличный выбор для примеров использования с высокой пропускной способностью и низкой задержкой, в которых требуется горизонтальное масштабирование, не ограниченное рамками одного инстанса;

— в базах данных NoSQL применяются различные модели данных, в том числе документные, графовые, поисковые, с использованием пар «ключ-значение» и хранением данных в памяти;

- в отличие от РСУБД производительность NoSQL зависит от размера кластера, задержки сети и вызывающего приложения, а не от дисковой подсистемы;

— гибкость в использовании встроенных языков.

Для осуществления успешной миграции необходимо произвести детальное сравнение двух СУБД, и определить возможности, совпадающие в обоих случаях. Под процессом миграции будем понимать процесс полного «переезда» с использования одной технологии на другую.

Целью данной работы является исследование миграции с реляционной базы данных на NewSQL решение на примере конкретной базы данных, структура которой была получена в ходе технического задания от организации, в которой работает один из авторов.

\section{Реляционная СУБД MySQL}

MySQL - это система управления реляционными базами данных. Она полностью открыта и распространяется по лицензии GNU GPL. В 2008 году компания Oracle купила компанию Sun Microsystems и завладела MySQL, в связи с чем появилась коммерческая лицензия. Также сообществом разработчиков были созданы различные ответвления кода. Самые известные на сегодняшний момент - это Percona Server и MariaDB.

За основу в MySQL взята реляционная модель данных, в которой главную роль играют таблицы и связи между ними. Такая модель данных имеет следующие преимущества:

- простота и доступность для понимания конечным пользователем;

- при проектировании реляционных баз данных применяются правила, которые базируются на математическом аппарате;

- отсутствие избыточности данных, благодаря процессу нормализации;

- при исполнении запросов к данным, навигация на физическом уровне происходит средствами самой СУБД.
Одним из самых важных преимуществ является идеология транзакционных систем ACID:

- атомарность (Atomicity) гарантирует, что никакая транзакция не будет выполнена частично;

- согласованность (Consistency) говорит о том, что успешная, зафиксированная транзакция сохраняет согласованность базы данных;

— изолированность (Isolation) говорит о том, что параллельные транзакции не оказывают влияния друг на друга;

— долговечность (Durability) обеспечивает сохранность изменений после успешных транзакции даже при проблемах на нижнем уровне.

Для манипулирования данными в MySQL используется язык структурированных запросов SQL. Данный язык не является процедурным. Изначально этот язык задумывался как средство работы конечного пользователя, но в ходе разработки он стал настолько сложным, что стал инструментом для разработчика. Операторы языка делятся на несколько групп:

- операторы определения (DDL), с помощью которых можно создавать, удалять и изменять объекты;

- операторы манипуляции (DML), c помощью которых производится выборка, обновление, удаление и изменение данных;

- операторы доступа к данным (DCL), с помощью которых можно выдавать права на определенные операции с объектами;

- операторы управления транзакциями (TCL), с помощью которых возможно применить или откатить транзакцию.

Следует отметить, что SQL реализует декларативную парадигму программирования: каждый оператор описывает только необходимое действие, а СУБД принимает решение о том, как его выполнить, т.е. планирует элементарные операции, необходимые для выполнения действия и выполняет их. Тем не менее, для эффективного использования возможностей SQL разработчику необходимо понимать то, как СУБД анализирует каждый оператор и создает его план выполнения.

СУБД MySQL обладает целым комплексом важных преимуществ перед другими реляционными системами. В частности, следует отметить такие качества как [2]:

- простота в использовании. MySQL достаточно легко инсталлируется, а наличие множества плагинов и вспомогательных приложений упрощает работу с базами данных;

- обширный функционал. Система MySQL обладает практически всем необходимым инструментарием, который может понадобиться в реализации практически любого проекта; 


\begin{tabular}{|c|c|c|c|c|c|c|}
\hline \multirow{4}{*}{ Impact Factor: } & ISRA (India) & $=3.117$ & SIS (USA) & $=0.912$ & ICV (Poland) & $=6.630$ \\
\hline & ISI (Dubai, UAE & $=0.829$ & РИНЦ (Russia & $=0.156$ & PIF (India) & $=1.940$ \\
\hline & GIF (Australia) & $=0.564$ & ESJI (KZ) & $=8.716$ & IBI (India) & $=4.260$ \\
\hline & JIF & $=1.500$ & SJIF (Morocce & $=5.667$ & OAJI (USA) & $=0.350$ \\
\hline
\end{tabular}

- безопасность. Система изначально создана таким образом, что множество встроенных функций безопасности в ней работают по умолчанию;

- масштабируемость. Являясь весьма универсальной СУБД, MySQL в равной степени легко может быть использована для работы и с малыми, и с большими объемами данных;

- по большей части система распространяется бесплатно;

- скорость. Высокая производительность системы обеспечивается за счет упрощения некоторых используемых в ней стандартов.

\section{NewSQL СУБД Tarantool}

Tarantool представляет собой решение с открытым исходным кодом, которое совмещает в себе систему управления базой данных и сервер приложений на языке Lua. История этой СУБД начинается в 2008. Одна из крупнейших российских IT компаний «Mail.ru» начала разработку собственной in-memory СУБД. Со временем Tarantool стал частью самого проекта Mail.ru и сейчас его используют для хранения и обработки динамического контента, например, сеансов пользователей, а также в качестве кэша для традиционных реляционных баз данных. Tarantool называют NewSQL решением. Термин NewSQL означает, что СУБД Tarantool включает в себя преимущества NoSQL вместе с поддержкой реляционных принципов ACID.

Основной движок этой базы данных работает по принципу in-memory, что означает, что работа с данными происходит в оперативной памяти. За счёт этого повышается производительность, пропускная способность, а также время отклика [3]. При работе с оперативной памятью присутствует риск потери данных, который может быть связан с отключением питания. Но журналы упреждающей записи и снэпшоты гарантируют сохранность ваших данных. Если количество обрабатываемых данных слишком велико, то в Tarantool есть движок Vinyl, который может хранить данные на диске.

Одним из самых больших плюсов является то, что весь код будет выполняться не на стороне клиента, а на встроенном сервере приложений.

Так как Tarantool является NoSQL решением, то здесь отсутствуют привычные нам в реляционной модели таблицы. Вместо этого Tarantool оперирует терминами спейс (от англ. Space) и тапл-кортеж (от англ. Tuple). Когда речь идет о хранении данных в Tarantool, то всегда существует хотя бы один спейс. Каждый спейс характеризуется уникальным числовым идентификатором и именем. При создании спейса можно указать движок Vinyl или Memtx. Спейсэто контейнер для кортежей, которому для работы нужен первичный индекс [4].
Кортежи похожи на строки в реляционной модели, а компоненты кортежа - поля, играют такую же роль, что и столбец, только с некоторыми замечаниями:

— поля кортежа могу представлять собой композитные структуры данных, такие как таблицы типа массива или ассоциативного массива;

— полям не обязательно присваивать имена, так как к ним можно обращаться по порядковому номеру.

Кортежи хранятся в виде массивов MsgPack. Это формат, очень схожий с общеизвестным форматом JSON, но он упаковывает данные в среднем на 15\% эффективнее. Так как Tarantool это база данных и сервер приложений одновременно, то работать одновременно надо с двумя наборами типов: типами языка программирования и типами внутреннего хранилища Tarantool - MsgPack.

Индексы - это совокупность значений и указателей. При создании индексу должно быть присвоено имя и автоматически сгенерированный ID. Любой спейс должен иметь первичный индекс, a вторичных может быть любое количество. Индексы в Tarantool могут быть многокомпонентными. Также есть возможность сделать индекс уникальным, то есть можно объявить, что дважды задавать одно значение невозможно.

Типы индексов в Tarantool по умолчанию Tree. Но также существуют и hash, bitset, rtree индексы. На рис.1 показан пример хранения данных в Tarantool.

Так же в Tarantool имеется такая структура как последовательность. Это генератор упорядоченных значений целых чисел. Для каждой последовательности можно указать начальное значение, шаг, минимальное и максимальное значение.

\section{Скриптовый язык Lua}

В отличие от языка SQL, который используется в традиционных реляционных базах данных, Tarantool на своем сервере приложений использует скриптовый язык Lua. Lua это процедурный динамически

типизированный модульный язык

с автоматическим управлением памятью. Включает базовые элементы для поддержки функционального

и объектного стилей программирования. Таким образом, Lua можно называть мультипарадигменным языком. Из-за того, что основным назначением Lua является встраивание, он имеет эффективные средства межъязыкового взаимодействия, ориентированные, главным образом, на вызов библиотек $\underline{\mathrm{Cn}}$ и на работу в Си-окружении. То 


\begin{tabular}{|c|c|c|c|c|c|c|}
\hline \multirow{4}{*}{ Impact Factor: } & ISRA (India) & $=3.117$ & SIS (USA) & $=0.912$ & ICV (Poland) & $=6.630$ \\
\hline & ISI (Dubai, UAI & $=0.829$ & РИНЦ (Russia & $=0.156$ & PIF (India) & $=1.940$ \\
\hline & GIF (Australia) & $=0.564$ & ESJI (KZ) & $=8.716$ & IBI (India) & $=4.260$ \\
\hline & JIF & $=1.500$ & SJIF (Morocco & $=5.667$ & OAJI (USA) & $=0.350$ \\
\hline
\end{tabular}

есть помимо пакетных функций можно писать собственные процедуры на языке С и вызывать их средствами Lua. Работать с данными в Tarantool посредством языка Lua сложнее, по сравнению с языком SQL в реляционной модели. Так как почти на каждое привычное действие в SQL требуется написать хранимую процедуру на языке Lua.

\section{Обзор аналогов Tarantool}

Что же касается представителей NoSQL, то из всего множества решений выберем решения тех компаний, на которые при создании Tarantool опирались разработчики "Mail.ru": RocksDB от Facebook и Redis. Прежде чем перейдём к обзору этих технологий, заметим, что они основаны на использовании технологии LSM-дерева.

RocksDB - $\quad$ встраиваемая высокопроизводительная key-value база данных. Это ответвление от LevelDB компании Google. Данная база данных оптимизирована под работу с высокоскоростными физическими хранилищами твердотельными накопителями. API представлен на множестве языков. Данное ПО распространяется свободно и имеет открытый исходный код. Несмотря на то, что это форк LevelDB, он имеет ряд преимуществ [5]:

- процесс compaction, который выполняет слияние файлов при переходе с одного уровня на другой, является многопоточным;
- команда insert так же многопоточна;

- уменьшено время удержания мьютекса;

- в memtable используется bloom filter;

- удаление конкретной записи по ключу.

Теперь рассмотрим NoSQL базу данных Redis [6]. Это резидентная система управления базами данных класса NoSQL. Под словом резидентная понимается in-memory хранение. Данная БД также имеет открытый исходный код и работает со структурами данных ключ-значение. В сравнении с Tarantool сразу же можно найти серьезные минусы [7]:

— Tarantool имеет полноценный сервер приложений Lua, что позволяет реализовать любую логику (рис. 2). A в Redis Lua служит для небольших, локальных задач;

— Tarantool поддерживает вторичные

— Tarantool не ограничен в размере данных, так как у него имеется дисковый движок Vinyl. A Redis может хранить данные только в памяти;

- с версии 2 Tarantool имеет поддержку SQL, в Redis такой возможности не предусмотрено;

- небольшим преимуществом можно считать команду поддержки, которая доступна всегда для связи в канале одного из мессенджеров.
TUPLE [1, 'Roxette']

TUPLE [2, 'Scorpions', 2015]

TUPLE [3, 'Ace of Base', 1993]

INDEX 'secondary'

KEY ['Roxette']

KEY ['Scorpions']

KEY ['Ace of Base']

Рис. 1. Пример хранения данных в Tarantool 


\begin{tabular}{|c|c|c|c|c|c|c|}
\hline \multirow{4}{*}{ Impact Factor: } & ISRA (India) & $=3.117$ & SIS (USA) & $=0.912$ & ICV (Poland) & $=6.630$ \\
\hline & ISI (Dubai, UAE & $=0.829$ & РИНЦ (Russia) & $=0.156$ & PIF (India) & $=1.940$ \\
\hline & GIF (Australia) & $=0.564$ & ESJI (KZ) & $=8.716$ & IBI (India) & $=4.260$ \\
\hline & JIF & $=1.500$ & SJIF (Morocco) & $=5.667$ & OAJI (USA) & $=0.350$ \\
\hline
\end{tabular}

TARANTOOL ENTERPRISE ARCHITECTURE

BI, APPLICATION, SERVICES

NEW SYSTEM

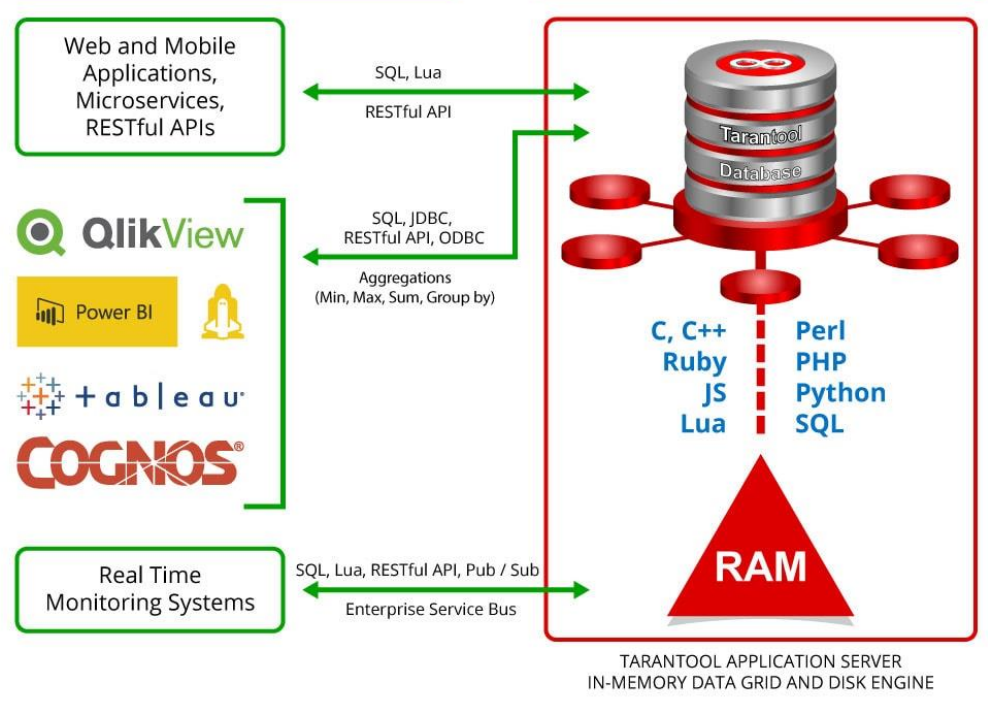

EXISTING SYSTEM
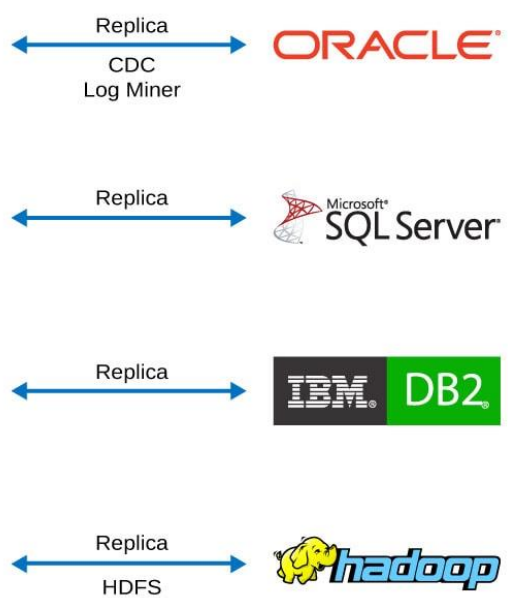

Рис. 2. Пример архитектуры с использованием Tarantool

\section{решений}

Обзор используемых технических

Для работы с MySQL помимо командной строки мы использовали MySQL Workbench визуальный инструмент для архитекторов, разработчиков и администраторов баз данных, который интегрирует в себе проектирование, моделирование, создание и эксплуатацию БД в единое окружение [8].

MySQL поддерживает несколько различных движков и также имеет возможность подключить сторонний. Основными поставляемыми движками являются InnoDB и MyISAM.

В нашем случае мы будем использовать InnoDB, так как он имеет ряд преимуществ [9]:

- присутствует поддержка транзакций;

- поддерживает внешние ключи;

- при работе с данными блокировки происходят на уровне строк, а не таблицы целиком;

- при смешанной нагрузке на таблицу или, иными словами, использовании команд DML в равной степени, работает быстрее, чем MyISAM;

— из-за поддержки транзакций есть возможность в случае сбоя восстановить базу по лог файлам.

Но, несмотря на очевидные преимущества, InnoDB также имеет и недостатки по сравнению с MyISAM:

- полнотекстовый поиск появился только начиная с версии 5.6.4, в то время как MyISAM всегда имел такую возможность;
— если при работе с базой сильно преобладают операции чтения, то работать будет быстрее MyISAM;

- файлы данных движка InnoDB занимают примерно в 1.5 раза больше места на диске;

- операция вставки данных происходит быстрее в MyISAM.

Хранение данных осуществляется с использованием такой структуры, как В-дерево. Это сбалансированное дерево, состоящее из блоков. При этом каждый блок содержит отсортированный список элементов, то есть пары ключ - значение. Для минимального представления дерева, рассмотрим такие операции, как поиск и обновление данных. Поиск осуществляется с вершины: если ключ будет обнаружен в корневом блоке, то поиск закончится, иначе мы перейдём в блок с наибольшим первым ключом или в самый правый блок. В случае неудачи мы будем двигаться по блокам вниз, пока не достигнем нужного. При использовании этой структуры предполагается, что блоки хранятся на дисковом хранилище и читаются целиком в оперативную память, иными словами за поиск считывается $\log _{\mathrm{B}}(\mathrm{N})$ блоков, где $\mathrm{N}$ - количество элементов в дереве. По сути, запись - аналогичная операция, которая начинается с поиска и обновляет значение в блоке.

Рассмотрим небольшой пример В-дерева (рис. 3). Пусть размер элемента 100 байт, а размер блока 4096 байт. Предположим, что мы обновляем элемент, тогда, работая с деревом посредством чтения и записи блоков, мы вынуждены прочитать блок в память, изменить 100 байт из 4096 и записать новый блок целиком на диск, получая в 


\begin{tabular}{|c|c|c|c|c|c|c|}
\hline \multirow{4}{*}{ Impact Factor: } & ISRA (India) & $=3.117$ & SIS (USA) & $=0.912$ & ICV (Poland) & $=6.630$ \\
\hline & ISI (Dubai, UAE & $=0.829$ & РИНЦ (Russia & $=0.156$ & PIF (India) & $=1.940$ \\
\hline & GIF (Australia) & $=0.564$ & ESJI (KZ) & $=8.716$ & IBI (India) & $=4.260$ \\
\hline & JIF & $=1.500$ & SJIF (Morocce & $=5.667$ & OAJI (USA) & $=0.350$ \\
\hline
\end{tabular}

40 раз больше записанных данных, чем реальный объём. Данный феномен в литературе называется «паразитной» записью\чтением.

Основной движок NoSQL СУБД Tarantool работает по принципу in-memory, что означает, что работа с данными происходит в оперативной памяти. За счёт этого повышается производительность, пропускная способность, а также время отклика. Но если возникает потребность в хранении большого количества данных, как в нашем случае, то приходит на помощь движок Vinyl, который хранит данные на диске.

Его ключевое отличие от стандартного движка InnoDB, который использует MySQL, в том, что он использует LSM дерево, как структуру хранения на диске, а не В-Tree дерево. Такое дерево имеет ряд преимуществ [10]:

- ключевое отличие LSM-деревьев от классических В-деревьев в том, что LSM хранит не данные, то есть ключи и значения, а операции с данными: вставки и удаления;

— в отличие от В-дерева, которое полностью хранится на диске и может частично кэшироваться в оперативной памяти, в LSM-дереве разделение между памятью и диском явно присутствует с самого начала;

- объём паразитных операций вводавывода при обновлении данных является одной из основных проблем, которую решают LSMдеревья;
— запись данных на различные уровни LSM дерева происходит в гораздо больших объёмах, чем блоки B-Tree, что позволяет эффективнее сжимать данные.

Для лучшего сравнения подробнее рассмотрим устройство LSM-дерева.

LSM призвано бороться с явлениями паразитной записи и чтения. В отличии от Вдерева, тут мы храним не сами данные, а операции с данными.

На рис. 4 мы видим, что элемент для операции вставки данных содержит дополнительный байт с кодом операции, также хранится log sequence number - значение последовательности, которое уникально идентифицирует каждую из операций. Итак, получаем, что наше дерево упорядочено по возрастанию ключа, а в пределах каждого из ключей по убыванию LSM.

В отличие от В-дерева, которое полностью хранится на диске и частично кэшируется, в LSM дереве разделение между памятью и диском присутствует изначально. Уровнем памяти является уровень L0, который ограничен объемом выделенной на него памяти. Когда объем этого уровня подходит к концу, то его содержимое записывается в файл на диске, сам уровень высвобождается для новых данных. Это операция называется Dump. На каждом уровне храним определенное количество dump, которые потом сливаются воедино, очищаясь от мусора. Пример работы с LSM-деревом показан на рис.5.

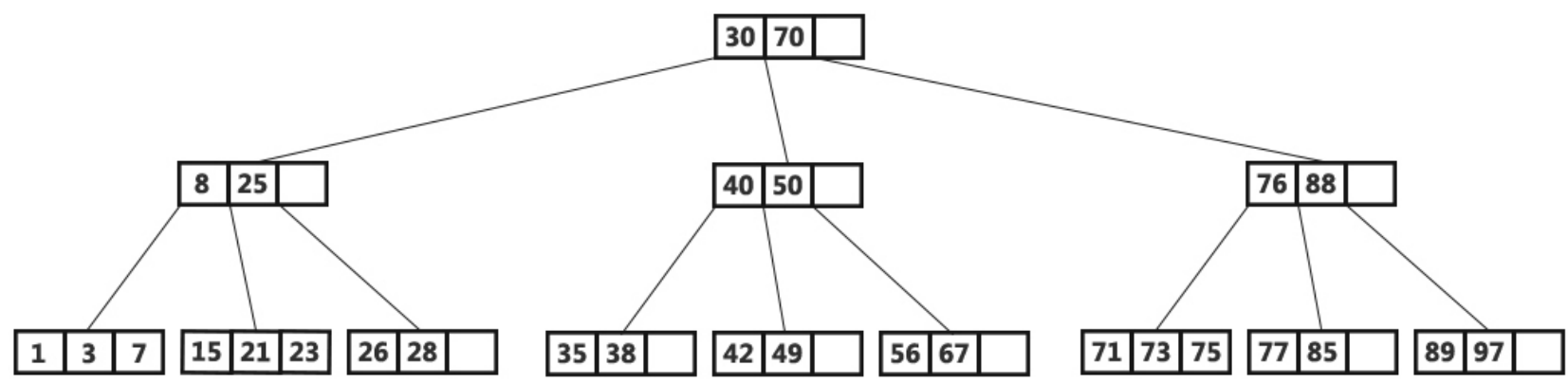

Рис. 3. Пример В-дерева

key Isn
\begin{tabular}{|c|c|c|c|}
\hline 1 & 176 & Op code & Value \\
\hline 1 & 53 & INSERLA & $2018-05-07$ 15:00:01 \\
\hline 2 & 174 & REPLACE & 2017-12-31 23:59:01 \\
\hline 3 & 175 & REPLACE & 2018-05 00:00 09:00 \\
\hline
\end{tabular}

Рис. 4. Устройство уровня в LSM 


\begin{tabular}{llllll} 
& ISRA (India) $=\mathbf{3 . 1 1 7}$ & SIS (USA) & $=\mathbf{0 . 9 1 2}$ & ICV (Poland) & $=\mathbf{6 . 6 3 0}$ \\
Impact Factor: & ISI (Dubai, UAE) $=\mathbf{0 . 8 2 9}$ & PUHL (Russia) $=\mathbf{0 . 1 5 6}$ & PIF (India) & $=\mathbf{1 . 9 4 0}$ \\
& GIF (Australia) $=\mathbf{0 . 5 6 4}$ & ESJI (KZ) & $=\mathbf{8 . 7 1 6}$ & IBI (India) & $=\mathbf{4 . 2 6 0}$ \\
& JIF & $\mathbf{1 . 5 0 0}$ & SJIF (Morocco) $=\mathbf{5 . 6 6 7}$ & OAJI (USA) & $\mathbf{0 . 3 5 0}$ \\
\hline
\end{tabular}

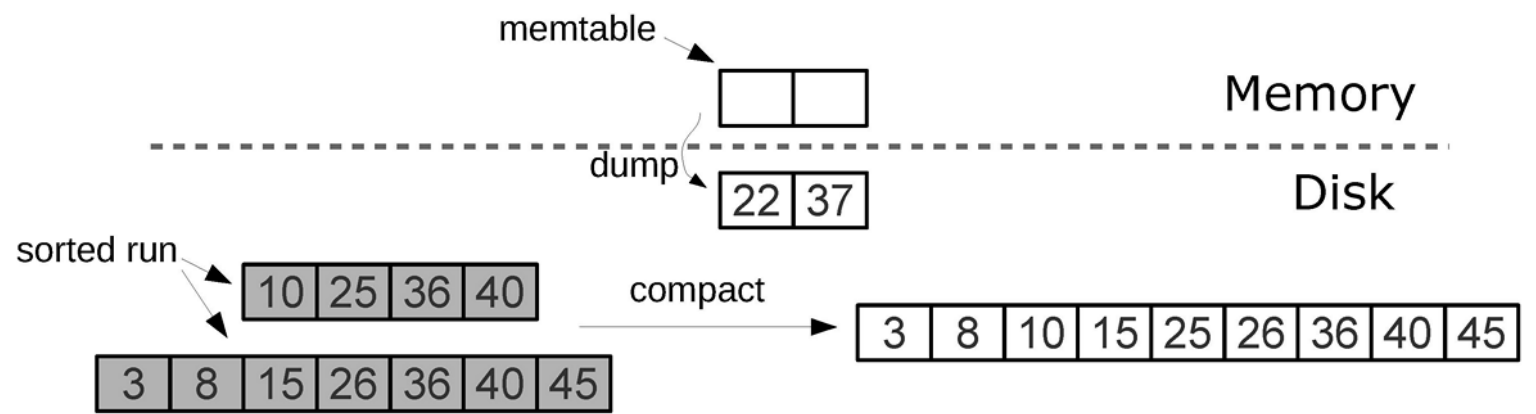

Рис. 5. Пример работы с LSM-деревом

\section{Проектирование баз данных}

Традиционно разработку баз данных начинают с определения ключевых сущностей из описания набора данных для хранения, а уже потом хранимые процедуры, триггеры и функции, которые нам понадобятся для работы. В нашем случае обе базы данных должны быть спроектированы в соответствии с предложенным техническим заданием.

По заданию основным источником данных являются четко структурированные текстовые документы формата * $\operatorname{csv}$ или * txt, содержащие 2 или 3 поля, разделенные определённым разделителем. В связи с требованиями нельзя разглашать то, что мы планируем хранить в базе данных, поэтому заменим данные на выдуманные. Итак, основные сущности, которые нам потребуются:

— картина;

- отдельная сущность под каждый вид хеша, для хэширования имени картины;

- сущность, в которую будет происходить первичная загрузка данных, так как далее нам потребуется правильное распределение по остальным сущностям.

Перечислим некоторые требование к схеме:

- каждое имя картины имеет собственные характеристики;

— для каждого имени картины может быть несколько видов хешей;

- сущность каждого вида хеша должна содержать ссылку на имя картины;

- набор характеристик картины может расширяться;

- не может быть нескольких одинаковых хешей одного типа для одной картины;

— при загрузке данных должна проверяться их корректность;

- все некорректные данные требуется хранить в отдельной сущности.

\section{Создание экземпляров на виртуальной} машине

Поскольку результаты тестирования зависят от настроек СУБД, для корректности приводим соответствующие настройки. В табл. 1 приводим конфигурацию для MySQL [11].

\section{Таблица 1. Содержание файла конфигурации my.cnf}

$\begin{array}{ll}\text { Название } & \text { Значение } \\ \text { query_cache_size } & \text { 32M }\end{array}$

query_cache_limit max_heap_table_size

$512 \mathrm{~K}$

$1 \mathrm{G}$

tmp_table_size

innodb_strict_mode
OFF
Описание

Определяет размер выделяемой памяти для кэша запросов. Не рекомендуется устанавливать слишком большое значение, так как кэш для определенной таблицы сбрасывается каждый раз при её изменении. Ограничение на кэшируемый элемент.

Максимальный размер для созданных пользователем таблиц, которые используют engine=Memory (хранятся не на диске, а в памяти).

Максимальный размер временной таблицы, которая будет создана в памяти (больше - на диске).

Если значение ON, то InnoDB возвращает ошибки вместо предупреждений (строгий режим SQL). Значение OFF по умолчанию, отключает этот режим. 


\begin{tabular}{|c|c|c|c|c|c|c|}
\hline \multirow{4}{*}{ Impact Factor: } & ISRA (India) & $=3.117$ & SIS (USA) & $=0.912$ & ICV (Poland) & $=6.630$ \\
\hline & ISI (Dubai, UAE & $=0.829$ & РИНЦ (Russia) & $=0.156$ & PIF (India) & $=1.940$ \\
\hline & GIF (Australia) & $=0.564$ & ESJI (KZ) & $=8.716$ & IBI (India) & $=4.260$ \\
\hline & JIF & $=1.500$ & SJIF (Morocco) & $=5.667$ & OAJI (USA) & $=0.350$ \\
\hline
\end{tabular}

\begin{tabular}{|c|c|c|}
\hline innodb_buffer_pool_size & $11 \mathrm{G}$ & Собственный буфер InnoDB для кэширования \\
\hline & & $\begin{array}{l}\text { индексов и данных. Рекомендуется } \\
\text { устанавливать значение равное } 70-80 \% \text { от всей } \\
\text { доступной оперативной памяти. }\end{array}$ \\
\hline innodb_buffer_pool_instances & 11 & $\begin{array}{l}\text { В ряде случаев InnoDB работает гораздо } \\
\text { эффективнее, если пул поделён на части } \\
\text { Стоит дробить пул, если размера пула } \\
\text { превышает } 2 \text { ГБ. }\end{array}$ \\
\hline innodb_data_file_path & ibdata1:10M:autoextend & $\begin{array}{l}\text { Размер индивидуального файла данных } \\
\text { InnoDB. }\end{array}$ \\
\hline innodb_file_per_table & ON & $\begin{array}{l}\text { InnoDB хранит каждую таблицу и ее индексы } \\
\text { в отдельном файле. }\end{array}$ \\
\hline innodb_log_file_size & $512 \mathrm{M}$ & $\begin{array}{l}\text { Эта опция влияет на скорость записи. Она } \\
\text { устанавливает размер лога операций (так } \\
\text { операции сначала записываются в лог, а потом } \\
\text { применяются к данным на диске). Чем больше } \\
\text { этот лог, тем быстрее будут работать записи } \\
\text { (т.к. их поместится больше в файл } \\
\text { лога). Файлов всегда 2. Увеличение этого } \\
\text { параметра может увеличить скорость записи, } \\
\text { но снизит скорость восстановления. }\end{array}$ \\
\hline innodb_lock_wait_timeout & 5 & $\begin{array}{l}\text { Время, которое ожидает транзакция в случае } \\
\text { обнаружения row-lock. }\end{array}$ \\
\hline innodb_autoinc_lock_mode & 2 & $\begin{array}{l}\text { Режим генерации автоинкремента. Значение } 2 \\
\text { не блокирует всю таблицу. Повышает } \\
\text { быстродействие, но небезопасен д } \\
\text { репликаций. }\end{array}$ \\
\hline innodb_flush_log_at_trx_commit & 1 & $\begin{array}{l}\text { Каждая операция будет записываться в лог } \\
\text { файл. Для успешного восстановления данных } \\
\text { в аварийном случае установить параметр в } 1 \\
\text { (Замедляет работу) }\end{array}$ \\
\hline innodb_flush_method & O_DIRECT & $\begin{array}{l}\text { Повышает надежность записи на диск } \\
\text { (Замедляет работу) }\end{array}$ \\
\hline innodb_file_format & Barracuda & $\begin{array}{l}\text { Формат файла для таблиц InnoDB, в отличие } \\
\text { от Antelope, поддерживает компрессию. }\end{array}$ \\
\hline
\end{tabular}

Создание экземпляра Tarantool также начинается с установки необходимого программного обеспечения. Далее мы можем воспользоваться интерактивным режимом, напрямую запустив службу tarantool, либо можем зайти в утилиту tarantoolctl для внешнего управления экземпляром.

Сервер Tarantool также имеет файл инициализации, с помощью которого

настраивается экземпляр. По сути, этот файл представляет собой скрипт языка Lua, в котором мы вызывает встроенный модуль Вох. Этот модуль содержит метод box.cfg \{\} , который в качестве аргументов принимает параметры сервера. В табл. 2 приводим пример конфигурации экземпляра Tarantool [12].

\section{Таблица 2. Параметры конфигурации Tarantool}

$\begin{array}{ll}\text { Название } & \text { Значение } \\ \text { listen } & 3301 \\ & \\ \text { Memtx_dir } & \text { 'memtxsnap' } \\ \text { Memtx_memory } & 128 * 1024 * 1024 \\ & (128 \mathrm{Mb})\end{array}$

\section{Описание}

Номер порта для чтения/записи данных или строка URI (унифицированный идентификатор pecypca)

Название директории, в которой будут храниться снепшоты(.snap)

Количество памяти, которое Tarantool выделяет для фактического хранения кортежей, в байтах. При достижении предельного значения запросы вставки INSERT или 


\begin{tabular}{|c|c|c|c|c|c|c|}
\hline \multirow{4}{*}{ Impact Factor: } & ISRA (India) & $=3.117$ & SIS (USA) & $=0.912$ & ICV (Poland) & $=6.630$ \\
\hline & ISI (Dubai, UAE & $=0.829$ & РИНЦ (Russia & $=0.156$ & PIF (India) & $=1.940$ \\
\hline & GIF (Australia) & $=0.564$ & ESJI (KZ) & $=8.716$ & IBI (India) & $=4.260$ \\
\hline & JIF & $=1.500$ & SJIF (Morocce & $=5.667$ & OAJI (USA) & $=0.350$ \\
\hline
\end{tabular}

Memtx_min_tuple_size

16(byte)

Memtx_max_tuple_size

$128 * 1024 * 1024$

(128Mb)

memtx_snap_io_rate_limit

nil

$\begin{array}{ll}\text { Vinyl_dir } & \text { 'vinylfile' } \\ \text { vinyl_memory } & 128 * 1024 * 1024 \\ & (128 \mathrm{Mb}) \\ \text { vinyl_cache } & 128 * 1024 * 1024 \\ & (128 \mathrm{Mb}) \\ \text { vinyl_max_tuple_size } & 128 * 1024 * 1024 \\ & (128 \mathrm{Mb})\end{array}$

vinyl_write_threads

5

vinyl_run_size_ratio

5

vinyl_run_count_per_leve

2

wal_dir

wal_max_size

'waldir'

$256 * 1024 * 1024$

(256Mb)

обновления UPDATE выполняться не будут, выдавая ошибку ER_MEMORY_ISSUE.

Размер наименьшего блока выделения памяти в байтах. Его можно уменьшить, если кортежи очень малого размера. Значение должно быть от 8 до 1048 280 включительно

Размер наибольшего блока выделения памяти в байтах для движка базы данных memtx. Его можно увеличить, если есть необходимость в хранении больших кортежей.

Уменьшение загрузки box.snapshot при выполнении операций вставки, обновления и удаления (INSERT/UPDATE/DELETE) путем установки предела скорости записи на диск - количества мегабайт в секунду.

Название директории, в которой будут храниться файлы дискового движка.

Максимальное количество байтов оперативной памяти, которые использует vinyl.

Размер кэша для движка базы данных vinyl в байтах. Размер кэша можно изменить динамически.

Размер наибольшего блока выделения памяти в байтах для движка базы данных vinyl. Его можно увеличить, если есть необходимость в хранении больших кортежей.

Максимальное количество потоков записи, которые vinyl может использовать в одновременных операциях, такие как ввод-вывод и компрессия.

Отношение размеров различных уровней журнальноструктурированного дерева со слиянием.

Максимальное количество забегов на уровень журнально-структурированного дерева со слиянием в vinyl'e.

Директория, где хранятся файлы журнала упреждающей записи (.xlog).

Максимальное количество байтов в отдельном журнале упреждающей записи. Если в результате запроса файл .xlog будет больше, чем указано в параметре wal_max_size, Tarantool создает другой WAL-файл - то же самое происходит, когда достигнуто количество строк в журнале, указанное B rows_per_wal.

\section{Разработка}

Основная проблема миграции заключается в том, что с одной стороны у нас реляционная модель и язык SQL, a с другой NoSQL и скриптовый язык Lua. Поэтому любое действие, которое мы можем реализовать привычными средствами языка SQL, нам придётся реализовывать на языке Lua.

Прямая миграция, которая подразумевала бы перенос схем, функций и самих данных в ходе данной работы не предусмотрена. Так как источник данных одинаковый, структура заранее известна, то попытаемся реализовать одинаковые схемы в обоих СУБД.

Для начала реализуем сами таблицы. Рассмотрим классическую реализацию на SQL на примере таблицы picture (рис. 6).

Аналогом таблицы в Tarantool является спейс. Создадим спейс picture (рис.7).

Как можно заметить из приведенного кода создания спейса, большим преимуществом Тарантула и относительной простотой повторения структуры реляционной базы данных является то, что у спейса есть формат, который очень похож на формат полей в РСУБД. На рис. 6 и 7 продемонстрирован лишь фрагмент кода. 


\begin{tabular}{|c|c|c|c|c|c|c|}
\hline \multirow{4}{*}{ Impact Factor: } & ISRA (India) & $=3.117$ & SIS (USA) & $=0.912$ & ICV (Poland) & $=6.630$ \\
\hline & ISI (Dubai, UAI & $=0.829$ & РИНЦ (Russia & $=0.156$ & PIF (India) & $=1.940$ \\
\hline & GIF (Australia) & $=0.564$ & ESJI (KZ) & $=8.716$ & IBI (India) & $=4.260$ \\
\hline & JIF & $=1.500$ & SJIF (Morocco & $=5.667$ & OAJI (USA) & $=0.350$ \\
\hline
\end{tabular}

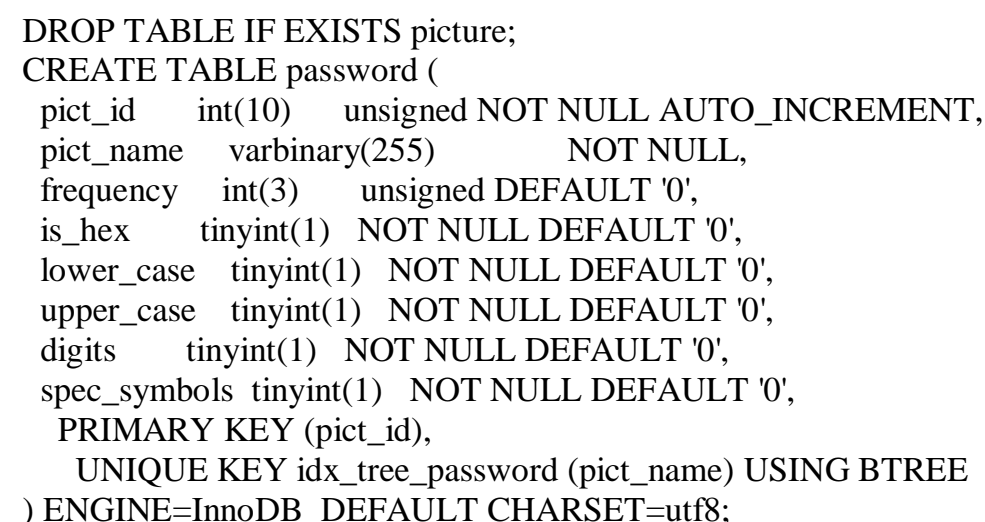

Рис. 6. Фрагмент кода создания таблицы на языке SQL

box.schema.space.create (picture, $\{$ engine='vinyl' $\}$ )

box.space.picture:format $(\{$

$\{$ name $=$ 'pict_id',type $=$ 'unsigned' $\}$,

$\{$ name $=$ 'pict_name',type $=$ 'string' $\}$,

$\{$ name $=$ 'frequency', type $=$ 'number' $\}$,

$\{$ name $=$ 'lower_case', type $=$ 'boolean' $\}$,

\{name = 'upper_case', type $=$ 'boolean' $\}$,

$\{$ name $=$ 'digits', type $=$ 'boolean' $\}$,

\})

$\{$ name $=$ 'symbols', type $=$ 'boolean' $\}$

box.schema.sequence.create('PictPK', $\{$ min=1,start=1,step=1 $\}$ )

box.space.picture:create_index $($ PictPK', $\{$ sequence $=$ 'PictPK',parts $=\{$ 'pict_id' $\}\})$

box.space.password:create_index('PictSecPictname', $\{$ parts $=\{$ 'pict_name' $\}\})$

\section{Рис. 7. Фрагмент кода создания спейса на языке LUA}

Что касается хранимых процедур, то они тоже сильно отличаются, приведем пример реализации одной из самых основных функции, которая реализует загрузку данных из текстового файла. В случае Mysql есть SQL команда, которая это делает (рис. 8).

В случаe Tarantool готовых процедур нет, есть различные модули - аналог подключаемых библиотек в обычных языках программирования. Для загрузки и чтения файла нам понадобится модуль csv и модуль fio. Далее самым простым способом построчно прочитаем файл и произведём вставку в спейс (рис. 9).

Как можно понять из кода, благодаря встроенному серверу приложений Lua мы можем получить некоторую гибкость в отличие от SQL. По окончанию работы команды SQL мы не видим сразу какого-то результата и не можем влиять на ход работы функции load data infile. Иными словами, если произойдёт какая-то ошибка, работа функции прервётся, и транзакция откатится. А в случаe Lua мы можем сделать обработку ошибок и, например, проверять корректность данных сразу.

Одним из самых трудных мест является операция соединения таблиц, если на языке SQL это всего лишь оператор, то в случае с Tarantool встроенной функции соединения спейсов нет.

Допустим, нам требуется из сущности для хранения промежуточного результата разделить хэши от имён картин. Ha SQL это выглядело бы так - рис.10. То есть мы просто указываем таблицы и оператор join, а в случаe Lua нам приходится реализовывать это вручную, проходя по спейсам внутри цикла (рис. 11) [10]. 


\begin{tabular}{llllll} 
& ISRA (India) $=\mathbf{3 . 1 1 7}$ & SIS (USA) & $=\mathbf{0 . 9 1 2}$ & ICV (Poland) & $=\mathbf{6 . 6 3 0}$ \\
Impact Factor: & ISI (Dubai, UAE) $=\mathbf{0 . 8 2 9}$ & PUHL (Russia) $=\mathbf{0 . 1 5 6}$ & PIF (India) & $=\mathbf{1 . 9 4 0}$ \\
& GIF (Australia) $=\mathbf{0 . 5 6 4}$ & ESJI (KZ) & $=\mathbf{8 . 7 1 6}$ & IBI (India) & $=\mathbf{4 . 2 6 0}$ \\
& JIF & $\mathbf{1 . 5 0 0}$ & SJIF (Morocco) $=\mathbf{5 . 6 6 7}$ & OAJI (USA) & $\mathbf{0 . 3 5 0}$ \\
\hline
\end{tabular}

load data infile 'filename' IGNORE into table table_ name fields terminated by ':' lines terminated by 'In' (hash_data,pict_name);

Рис. 8. Функция загрузки из текстового файла на языке SQL

function loadcsv(path)

local csv= require("csv")

local fio =require("fio")

local file = fio.open $($ path, $\{$ "O_RDONLY" $\})$

start_time $=$ os.clock ()

for _, tuple in csv.iterate(file, $\{$ delimiter=":",quote_char="ไr" $\})$ do

box.space.tmptab:insert \{box.sequence.TmptabPK:next(),tuple[1],'nil',tuple[2]\}

end

end_time $=$ os.clock ()

print(end_time-start_time)

end

Рис. 9. Функция вставки данных из файла на языке Lua

INSERT IGNORE INTO storage.md5(hash_data,pict_id,murmur)

SELECT hash_data, pict_id,murmur_hash(hash_data) FROM tmp_tab join picture $\mathrm{p}$

ON pict=p.pict WHERE pict IS NOT NULL and is_hex $=0$;

Puc. 10. SQL join

function spread_md5()

local data $=\{\}$

local psdata $=\{\}$

start_time $=$ os.clock ()

data=box.space.tmptab:select()

psdata=box.space.picture:select ()

for _, tupleTMP in pairs(data) do

for _, tuplePSW in pairs(psdata) do

if (tupleTMP[4]==tuplePSW[2]) then

box.space.md5:insert(box.tuple.new(box.sequence.Md5PK:next(),tupleTMP[2],tuplePSW[1],0))

end

end

end

end_time $=$ os.clock ()

print(end_time-start_time)

Pис. 11. Lua join

Подобным образом, мы создали все таблицы и спейсы и реализовали все требующиеся функции.

\section{Экспериментальное исследование эффективности}

По техническому заданию сравнение эффективности требуется производить по таким параметрам, как скорость вставки данных и скорость запросов с использованием јоin. Также важным критерием является сравнение занимаемого места при одном и том же наборе данных, а также размер индексов. В связи с некоторыми ограничениями, использовать вычислительные мощности предприятия запрещено. Поэтому создадим собственную виртуальную машину со след параметрами:

- O3У $128 \mathrm{Mb}$

- HDD 5600 оборотов \мин;

- Intel Core i5-6200U 2.40Ghz; CentOS 7.4.

- виртуальная машина под управлением

Скорость вставки данных будем проверять при первичной загрузке данных. Для начала тестирования требуется подготовить исходные файлы различного размера. В табл.3 приведём список файлов. На рис.12 рассмотрен пример информации, которая содержится в исходных файлах. 


\begin{tabular}{|c|c|c|c|c|c|c|}
\hline \multirow{4}{*}{ Impact Factor: } & ISRA (India) & $=3.117$ & SIS (USA) & $=0.912$ & ICV (Poland) & $=6.630$ \\
\hline & ISI (Dubai, UAE & $=0.829$ & РИНЦ (Russia) & $=0.156$ & PIF (India) & $=1.940$ \\
\hline & GIF (Australia) & $=0.564$ & ESJI (KZ) & $=8.716$ & IBI (India) & $=4.260$ \\
\hline & JIF & $=1.500$ & SJIF (Morocco) & $=5.667$ & OAJI (USA) & $=0.350$ \\
\hline
\end{tabular}

Таблица 3. Список исходных файлов

\begin{tabular}{|l|l|l|}
\hline Имя файла & $\begin{array}{l}\text { Размер } \\
\text { (Мб) }\end{array}$ & Количество строк \\
\hline testmd51 & 1,40 & 31187 \\
\hline testmd52 & 1,20 & 27167 \\
\hline testmd53 & 0,02 & 508 \\
\hline testmd54 & 8,30 & 191557 \\
\hline testmd55 & 3,00 & 67644 \\
\hline testmd56 & 2,80 & 64130 \\
\hline testmd57 & 0,04 & 1021 \\
\hline testmd58 & 0,26 & 6389 \\
\hline testmd59 & 0,55 & 12652 \\
\hline testmd510 & 4,20 & 95805 \\
\hline testmd511 & 4,70 & 110333 \\
\hline testmd512 & 63,80 & 1501105 \\
\hline testmd513 & 9,20 & 222345 \\
\hline testmd514 & 17,70 & 412309 \\
\hline testmd515 & 151,80 & 3528719 \\
\hline testmd516 & 35,90 & 813760 \\
\hline testmd517 & 10,30 & 243675 \\
\hline
\end{tabular}

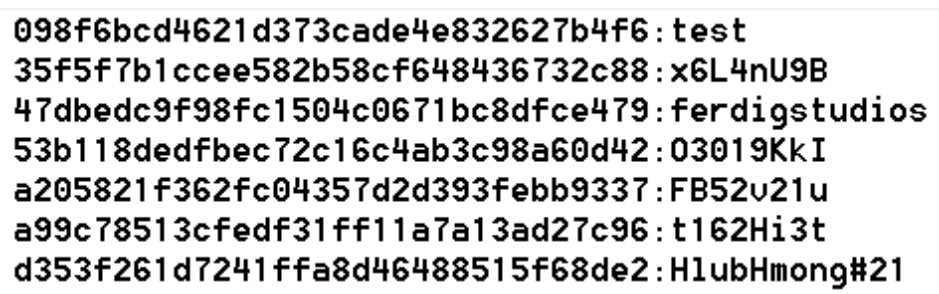

Рис. 12. Пример исходного файла

Начнём вставку данных в MySQL, для этого будем использовать заранее подготовленный скрипт, фрагмент которого был представлен на рис. 8. Замер времен работы скрипта будем проводить с помощью команды date операционной системы CentOS. После выполнения вставки данных из всех исходных файлов, занесем результаты в табл.4.

Теперь выполним загрузку данных в Tarantool. Для этого будем использовать скрипт, фрагмент которого представлен на рис.9. В ходе загрузки с использованием этого скрипта получаются очень плохие результаты, так как команда для вставки insert делает скрытые чтения перед вставкой, что увеличивает время вставки и не дает никакого преимущества перед MySQL. Поэтому нам пришлось внести изменения в скрипт, заменив команду insert на replace, которая работает на порядок быстрее. На рис.13. представлен исправленный скрипт для вставки из текстового файла.

Таблица 4. Результаты вставки в MySQL

\begin{tabular}{|l|l|}
\hline Строки & Время (сек) \\
\hline 508 & 0,04 \\
\hline 1021 & 0,06 \\
\hline 6389 & 0,35 \\
\hline 12652 & 0,63 \\
\hline 27176 & 0,83 \\
\hline 31187 & 1,17 \\
\hline 64130 & 1,74 \\
\hline 67644 & 2,14 \\
\hline 95805 & 2,74 \\
\hline 110333 & 2,88 \\
\hline
\end{tabular}




\begin{tabular}{|c|c|c|c|c|c|c|}
\hline \multirow{4}{*}{ Impact Factor: } & ISRA (India) & $=3.117$ & SIS (USA) & $=0.912$ & ICV (Poland) & $=6.630$ \\
\hline & ISI (Dubai, UAE & $=0.829$ & РИНЦ (Russia & $=0.156$ & PIF (India) & $=1.940$ \\
\hline & GIF (Australia) & $=0.564$ & ESJI (KZ) & $=8.716$ & IBI (India) & $=4.260$ \\
\hline & JIF & $=1.500$ & SJIF (Morocce & $=5.667$ & OAJI (USA) & $=0.350$ \\
\hline
\end{tabular}

\begin{tabular}{|l|l|}
191557 & 4,79 \\
\hline 222345 & 5,02 \\
\hline 243675 & 5,44 \\
\hline 412309 & 8,91 \\
\hline 813760 & 20,21 \\
\hline 1501105 & 48,65 \\
\hline 3528719 & 282,45 \\
\hline
\end{tabular}

function loadcsv(path)

local csv= require("csv")

local fio =require("fio")

local file = fio.open $($ path, $\{$ "O_RDONLY" $\})$

start_time $=$ os.time ()

for _, tuple in csv.iterate(file, $\{$ delimiter=":",quote_char="|r",chunk_size=4096*4096\}) do

box.space.tmptab:replace\{tuple[1],'nil',tuple[2]\}

end

end_time $=$ os.time ()

print(os.date("\%H:\%M:\%S",(end_time-start_time)-10800))

end

\section{Рис. 13. Фрагмент нового скрипта для вставки данных}

Замер времени работы в данном случае проводится с помощью команды os.time() языка Lua, которая эквивалентна команде date операционной системы CentOS. После обработки всех файлов получим результаты, представленные в табл.5. Для наглядности сравнения построим графики на основе полученных результатов (рис. 14).

Таблица 5. Результаты вставки в Tarantool

\begin{tabular}{|l|l|}
\hline Строки & Время (сек) \\
\hline 508 & 0,00 \\
\hline 1021 & 0,00 \\
\hline 6389 & 0,00 \\
\hline 12652 & 0,00 \\
\hline 27176 & 0,02 \\
\hline 31187 & 0,01 \\
\hline 64130 & 0,03 \\
\hline 67644 & 0,02 \\
\hline 95805 & 0,03 \\
\hline 110333 & 0,03 \\
\hline 191557 & 0,04 \\
\hline 222345 & 0,08 \\
\hline 243675 & 0,06 \\
\hline 412309 & 0,11 \\
\hline 813760 & 0,22 \\
\hline 1501105 & 0,38 \\
\hline 3528719 & 112,00 \\
\hline
\end{tabular}




\begin{tabular}{llllll} 
& ISRA (India) $=\mathbf{3 . 1 1 7}$ & SIS (USA) & $=\mathbf{0 . 9 1 2}$ & ICV (Poland) & $=\mathbf{6 . 6 3 0}$ \\
Impact Factor: & ISI (Dubai, UAE) $=\mathbf{0 . 8 2 9}$ & PUHL (Russia) $=\mathbf{0 . 1 5 6}$ & PIF (India) & $=\mathbf{1 . 9 4 0}$ \\
& GIF (Australia) $=\mathbf{0 . 5 6 4}$ & ESJI (KZ) & $=\mathbf{8 . 7 1 6}$ & IBI (India) & $=\mathbf{4 . 2 6 0}$ \\
& JIF & $\mathbf{1 . 5 0 0}$ & SJIF (Morocco) $=\mathbf{5 . 6 6 7}$ & OAJI (USA) & $\mathbf{0 . 3 5 0}$ \\
\hline
\end{tabular}

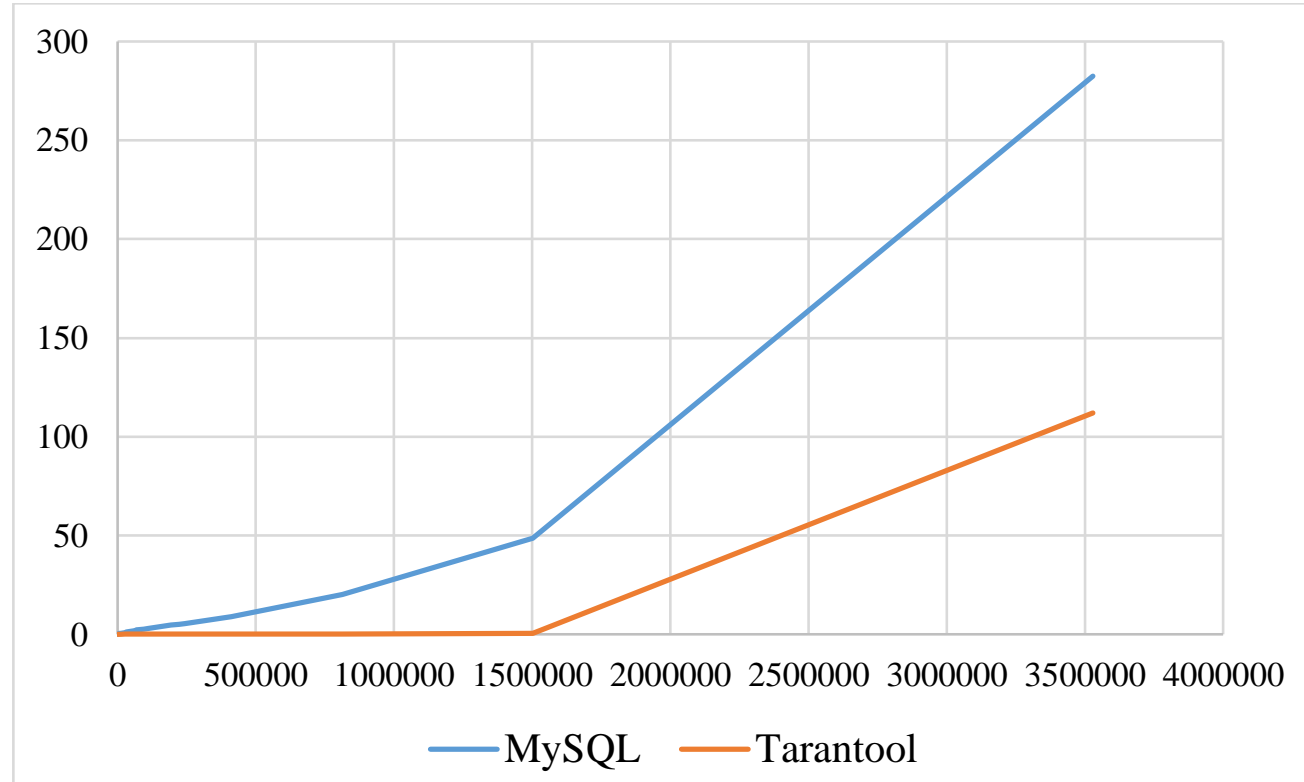

Рис. 14. График сравнения скорости вставки данных (по оси ординат время в секундах, по оси абсцисс-количество строк)

Следующий важный момент, который необходимо учесть по техническому заданию во время миграции - размеры данных и индексов в каждой СУБД.

Для просмотра размера данных в спейсе и размера индекса, в Tarantool есть команда bsize(), вызываемая при обращении к объекту. В случае
MySQL нам требуется сделать запрос к внутреннему словарю данных (рис.15).

При выполнении вставки данных из всех исходных файлов будем измерять размер индексов, размер самих данных, суммарный размер и отношение суммарного размера к размеру исходного файла. Результат представим в табл.6.

SELECT table_schema,table_name,round ((data_length)/1024/1024,1) 'data in MB', round((index_length)/1024/1024,1) 'ind ind MB'

FROM information_schema.tables where table_schema = 'STORAGE' ;

Рис. 15. Запрос размера данных и индексов.

Таблица 6. Размер данных и индексов вставленных данных.

\begin{tabular}{|l|l|l|l|l|l|l|}
\hline & MySQL & Tarantool & MySQL & Tarantool & MySQL & Tarantool \\
\hline $\begin{array}{l}\text { Размер файла } \\
\text { (Мб) }\end{array}$ & \multicolumn{2}{|l|}{ Размер индекса (Мб) } & \multicolumn{2}{l|}{ Размер данных (Мб) } & \multicolumn{2}{l|}{ Суммарный размер(Мб) } \\
\hline 0,02 & 0,06 & 0,05 & 0,05 & 0,04 & 0,11 & 0,09 \\
\hline 0,04 & 0,07 & 0,05 & 0,09 & 0,08 & 0,16 & 0,13 \\
\hline 0,26 & 0,50 & 0,11 & 0,30 & 0,48 & 0,80 & 0,59 \\
\hline 0,55 & 1,50 & 0,17 & 1,50 & 0,98 & 3,00 & 1,15 \\
\hline 1,20 & 3,20 & 0,28 & 2,20 & 2,12 & 5,40 & 2,40 \\
\hline 1,40 & 3,50 & 0,31 & 2,50 & 2,42 & 6,00 & 2,74 \\
\hline 2,80 & 7,50 & 0,67 & 4,50 & 4,95 & 12,00 & 5,63 \\
\hline 3,00 & 6,50 & 0,64 & 5,50 & 5,31 & 12,00 & 5,95 \\
\hline
\end{tabular}




\begin{tabular}{|c|c|c|c|c|c|c|}
\hline \multirow{4}{*}{ Impact Factor: } & ISRA (India) & $=3.117$ & SIS (USA) & $=0.912$ & ICV (Poland) & $=6.630$ \\
\hline & ISI (Dubai, UAE & $=0.829$ & РИНЦ (Russia) & $=0.156$ & PIF (India) & $=1.940$ \\
\hline & GIF (Australia) & $=0.564$ & ESJI (KZ) & $=8.716$ & IBI (India) & $=4.260$ \\
\hline & JIF & $=1.500$ & SJIF (Morocco & $=5.667$ & OAJI (USA) & $=0.350$ \\
\hline
\end{tabular}

\begin{tabular}{|l|l|l|l|l|l|l|}
4,20 & 11,50 & 0,95 & 7,50 & 7,46 & 19,00 & 8,41 \\
\hline 4,70 & 12,60 & 1,20 & 8,50 & 8,48 & 21,10 & 9,68 \\
\hline 8,30 & 17,60 & 1,75 & 13,50 & 14,83 & 31,10 & 16,58 \\
\hline 9,20 & 16,60 & 2,05 & 15,50 & 16,82 & 32,10 & 18,86 \\
\hline 10,30 & 25,60 & 2,45 & 16,50 & 18,62 & 42,10 & 21,07 \\
\hline 17,70 & 32,60 & 3,94 & 28,60 & 31,81 & 61,20 & 35,75 \\
\hline 35,90 & 74,00 & 1,13 & 57,60 & 50,60 & 131,60 & 51,74 \\
\hline 63,80 & 71,80 & 16,13 & 94,60 & 115,21 & 166,40 & 131,34 \\
\hline 151,80 & 407,00 & 27,44 & 241,80 & 255,97 & 648,80 & 283,41 \\
\hline
\end{tabular}

По результатам можно увидеть, что в среднем размер данных в Tarantool превышает исходный размер в 2 раза, а размер данных в MySQL в среднем превышает размер исходных данных в 4 раза. Это обусловлено тем, что размеры индексов в СУБД MySQL очень велики.

Рассмотрим на небольшом примере, как этого можно избежать. Размеры индексов по текстовым полям достаточно большие, соответственно и поиск по такому индексу будет долгим. В нашем случае таким текстовым полем является хэш значение, которое состоит из 32 символов. Для уменьшения величины индексов одним из выходов может быть введение альтернативного уникального значения, по которому может быть построен индекс. Поэтому в нашем случае мы можем использовать Murmur hash [13]. Им можно покрыть множество в $2^{64}$ элементов. Этот хэш числовой, поэтому при хранении использует меньше места, чем строки из 32 символов.

Для наглядности отличия размеров вставленных данных от исходных построим график. На графике синим цветом обозначим MySQL, а оранжевым - Tarantool (рис. 16).

Последним важным тестом является скорости выполнения операций с использованием соединения таблиц. В нашем случае для тестирования выберем сценарий из технического задания, в котором нам требуется из таблицы для первичной вставки данных отделить в разные сущности хэши и названия картин, при этом для каждого хэша сохранить ссылку на название для ссылочной целостности. Результаты представим в табл.7.

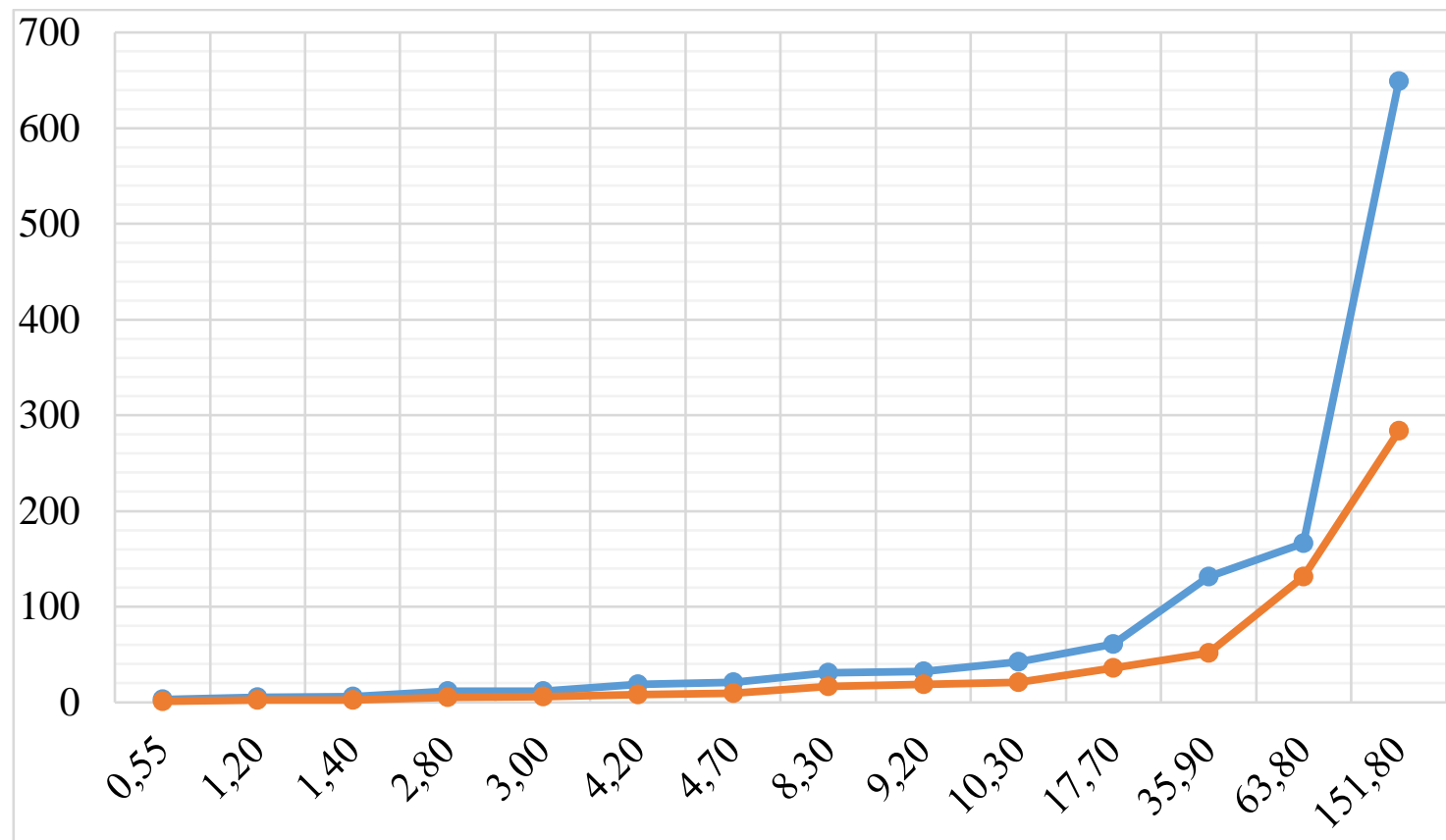

Рис. 16. Размеры вставленных данных (по оси ординат - суммарный размер в Мб, по оси абсцисс размер файла в Мб) 


\begin{tabular}{|c|c|c|c|c|c|c|}
\hline \multirow{4}{*}{ Impact Factor: } & ISRA (India) & $=3.117$ & SIS (USA) & $=0.912$ & ICV (Poland) & $=6.630$ \\
\hline & ISI (Dubai, UAE & $=0.829$ & РИНЦ (Russia & $=0.156$ & PIF (India) & $=1.940$ \\
\hline & GIF (Australia) & $=0.564$ & ESJI (KZ) & $=8.716$ & IBI (India) & $=4.260$ \\
\hline & JIF & $=1.500$ & SJIF (Morocce & $=5.667$ & OAJI (USA) & $=0.350$ \\
\hline
\end{tabular}

Таблица 7. Результаты с операцией вставки.

\begin{tabular}{|l|l|l|}
\hline Строки & MySQL (сек) & Tarantool (сек) \\
\hline 508 & 0,06 & 0 \\
\hline 1021 & 0,11 & 0,01 \\
\hline 6389 & 0,68 & 39 \\
\hline 12652 & 1,18 & 181 \\
\hline 27176 & 2,04 & 988 \\
\hline 31187 & 3,04 & 1723 \\
\hline 64130 & 4,46 & $!$ \\
\hline 67644 & 5,13 & $!$ \\
\hline 95805 & 7,16 & $!$ \\
\hline 110333 & 8,69 & $!$ \\
\hline 191557 & 15,79 & $!$ \\
\hline 222345 & 21,06 & $!$ \\
\hline 243675 & 22,17 & $!$ \\
\hline 412309 & 43,13 & $!$ \\
\hline 813760 & 350,95 & $!$ \\
\hline 1501105 & 362,47 & $!$ \\
\hline 3528719 & 840,35 & $!$ \\
\hline
\end{tabular}

По результатам использования операций с соединением таблиц мы можем увидеть, что это единственный тест, в котором Tarantool уступил по быстроте MySQL. Помимо этого невозможно было до конца провести тест из-за того, что время выполнения слишком велико. Это обусловлено тем, что для выполнения операции соединения таблиц в Tarantool была реализована функция с вложенным циклом, проходящим по обоим таблицам для поиска совпадения по определенному ключу. Это подразумевает сложность алгоритма $\mathrm{n}^{2}$.

Подводя итоги тестов, можно с уверенностью сказать, что миграция на СУБД Tarantool в рамках поставленной задачи успешна. Единственным моментом, который нуждается в переработке, является реализация соединения таблиц посредством языка Lua. B организации будет предложен пересмотр схемы баз данных для решения возникших проблем.

\section{Заключение}

В результате выполнения данной работы была произведена миграция из MySQL в Tarantool. В итоге были получены две схемы базы данных, способные хранить набор данных, обозначенный по техническому заданию. По результатам тестирования можно сделать вывод, что миграция на Tarantool в рамках задач, которые поставлены в техническом задании, принесёт значительный прирост производительности. Вдобавок, если учесть тот факт, что на предприятии планируется использовать целый кластер и технологию репликации, то можно сделать вывод, что это уменьшит число рабочих машин, которые мы бы использовали в случае с MySQL, так как экземпляры Tarantool гораздо эффективнее.

В перспективах для улучшения работы требуется провести более детальный анализ работы движков баз данных. Особое внимание стоит уделить схеме данных, так как если определённым способом её изменить, то получится убрать недостаток, который был выявлен в ходе тестирования операций соединения таблиц. Вдобавок, требуется более детально разобрать влияние каждого из параметров для настройки сервера баз данных, чтобы достичь максимальной производительности и правильно использовать предоставленные ресурсы рабочих машин. Также для комфорта работы предлагается реализовать графический интерфейс.

\section{References:}

1. (n.d.). Chto takoe bazy dannykh NoSQL? [Elektronnyy dokument] Retrieved July 08, 2019, from https://aws.amazon.com/ru/nosql/
2. (n.d.). Sistema upravleniya bazami dannykh MySQL [Elektronnyy dokument] Retrieved July 208, 2019 , from 


\begin{tabular}{llllll} 
& ISRA (India) $=\mathbf{3 . 1 1 7}$ & SIS (USA) & $=\mathbf{0 . 9 1 2}$ & ICV (Poland) & $=\mathbf{6 . 6 3 0}$ \\
Impact Factor: & ISI (Dubai, UAE) $=\mathbf{0 . 8 2 9}$ & PUHL (Russia) $=\mathbf{0 . 1 5 6}$ & PIF (India) & $=\mathbf{1 . 9 4 0}$ \\
& GIF (Australia) $=\mathbf{0 . 5 6 4}$ & ESJI (KZ) & $=\mathbf{8 . 7 1 6}$ & IBI (India) & $=\mathbf{4 . 2 6 0}$ \\
& JIF & $\mathbf{1 . 5 0 0}$ & SJIF (Morocco) $=\mathbf{5 . 6 6 7}$ & OAJI (USA) & $\mathbf{0 . 3 5 0}$ \\
\hline
\end{tabular}

https://depix.ru/articles/sistema_upravleniya_ba zami_dannyh_mysql

3. (n.d.). Za schet chego Tarantool takoy optimal'nyy? [Elektronnyy dokument] Retrieved July $\quad 08, \quad 2019, \quad$ from https://habr.com/ru/company/olegbunin/blog/340062/

4. (n.d.). Rukovodstvo pol'zovatelya Tarantool [Elektronnyy dokument] Retrieved July 08, 2019, from https://www.tarantool.io/ru/doc/2.1/book/

5. (n.d.). RocksDB Features [Elektronnyy dokument] Retrieved July 08, 2019, from https://github.com/facebook/rocksdb/wiki/Featu res-Not-in-LevelDB

6. (n.d.). Sravnivaem Tarantool $\mathrm{s}$ Redis i Memcached [Elektronnyy dokument] Retrieved July $\quad 08, \quad 2019, \quad$ from https://habr.com/ru/company/mailru/blog/35276 $\underline{0 /}$

7. (n.d.). Tarantool vs Redis [Elektronnyy dokument] Retrieved July 08, 2019, from https://hackernoon.com/tarantool-vs-redis38a4041cc4bc

8. (n.d.). MySQL Workbench Enhanced Data Migration [Elektronnyy dokument] Retrieved
July $\quad 08, \quad 2019, \quad$ from https://www.mysql.com/products/workbench/

9. (n.d.). Sravnenie InnoDB i MyISAM [Elektronnyy dokument] Retrieved July 08, 2019, from https://blog.programs74.ru/compareof-innodb-and-myisam-engines/

10. (n.d.). Khranenie dannykh na vinile [Elektronnyy dokument] Retrieved July 08, 2019, from https://habr.com/ru/company/mailru/blog/35821 $\underline{0 / \# 8}$

11. (n.d.). InnoDB Startup Options and System Variables [Elektronnyy dokument] Retrieved July 08, 2019, from https://dev.mysql.com/doc/refman/8.0/en/innod b-parameters.html

12. (n.d.). Spravochnik po nastroyke Tarantool [Elektronnyy dokument] Retrieved July 08, 2019 , from https://www.tarantool.io/ru/doc/1.10/reference/ configuration

13. (n.d.). MurMurHash [Elektronnyy dokument] Retrieved July 08, 2019, from https://ru.wikipedia.org/wiki/MurmurHash2 